\title{
Gambling by Young Adults in the UK During COVID-19 Lockdown
}

\author{
Alan Emond ${ }^{1}$ (D) $\cdot$ Agnes Nairn ${ }^{2} \cdot$ Sharon Collard ${ }^{3} \cdot$ Linda Hollén $^{1}$
}

Accepted: 20 April 2021 / Published online: 17 May 2021

(c) Springer Science+Business Media, LLC, part of Springer Nature 2021

\begin{abstract}
Gambling is a common activity amongst young adults in the UK, and was a behavior of interest during the early mitigation against COVID-19 (first lockdown). The Avon Longitudinal Study of Parents and Children (ALSPAC) was used to investigate attitudes, moods and behavior during lockdown in England. ALSPAC participants were invited to complete online questionnaires in May 2020, including a set of questions about frequency of gambling and gambling activities which had been asked three years previously. Mental health and wellbeing data and alcohol use were also collected as part of lockdown questionnaires. Gambling questions were completed by 2632 young adults, $71 \%$ female, with a mean age of 27.8 years. Overall, gambling frequency reduced during lockdown for both males and females, but more males engaged in regular (weekly) gambling. Gambling activities became more restricted compared to previous reports, but online gambling (e.g. online poker, bingo, casino games) was more frequent. Previous gambling behaviour predicted gambling frequency during lockdown. No associations were apparent between gambling frequency and measures of mental health and well-being. Heavy alcohol use was strongly linked with regular gambling during lockdown. Gamblers were more than twice as likely as non-gamblers to have experienced financial difficulties pre-COVID, but gambling frequency was not related to employment status during lockdown. Online gambling increased during lockdown, whilst offline gambling activities decreased in frequency. A small minority of regular weekly gamblers, who tended to be male and heavy users of alcohol, participated in a wide range of online and offline gambling activities.
\end{abstract}

Keywords Gambling · Lockdown · COVID-19 · ALSPAC

Alan Emond

alan.emond@bristol.ac.uk

1 Centre for Academic Child Health, Bristol Medical School, University of Bristol, 1-5 Whiteladies Road, Bristol BS8 1NU, UK

2 School of Management, Faculty of Social Science and Law, University of Bristol, Bristol, UK

3 School of Geographical Sciences, University of Bristol, Bristol, UK 


\section{Introduction}

In the UK, gambling is a common activity amongst young adults. In the Health Survey for England in 2018 (NHS Digital, 2019), 57\% of adult men and 54\% of adult women reported gambling in the past year. In the age range 16-24 years, $45 \%$ of men and $33 \%$ of women reported gambling in the past year, and $20 \%$ of men and $2 \%$ of women gambled online. The increased availability of gambling and the expansion of opportunities to gamble online have led to increases in the number of young people who gamble on a regular basis (Bray et al 2014; Delfabbro et al., 2014; Killick \& Griffiths, 2019; Gambling Commission, 2019).

Mitigation strategies against COVID-19 resulted in a comprehensive set of restrictions on normal life in the UK (termed 'lockdown') from 23rd March until the beginning of July 2020. During this period, many people were confined to their homes, with impacts on their physical and mental health and personal relationships. To capture the impact of lockdown, it was important for longitudinal studies to mount 'real- time' data collection and given its prevalence in the UK population (NHS Digital, 2019), gambling was one important behavior to investigate. During lockdown physical gambling premises were required to shut, but national lottery tickets and scratchcards could still be purchased from stores (as well as online), and online gambling continued as before, although in-game betting was limited by the lack of live sport.

The Avon Longitudinal Study of Parents and Children (ALSPAC), a three generation cohort based in the SW of England (http://www.bristol.ac.uk/alspac), was able to collect repeat data quickly during lockdown using an existing infrastructure for online data collection (Northstone et al., 2020). The young people in ALSPAC had previously been asked about their gambling activities at the age of 24 years as part of a longitudinal investigation of gambling in the transition from adolescence to young adulthood (Hollén et al., 2020). Aged 24 years, $66 \%$ reported gambling in the last year, with a heavy male bias in the $17 \%$ of participants admitting regular weekly gambling. The same gambling questions were asked of the young adults (mean age 27.8 years) in the online questionnaire during lockdown. Previous research suggests that young adult men are a group particularly likely to use the internet for gambling (Calado et al 2017; Emond et al., 2020), and those young men that gamble regularly have a higher rate of mental health problems (Petry \& Weinstock, 2007) and of abusing alcohol (French et al., 2008) — so this group were of special interest for this gambling research carried out in lockdown. The hypothesis to be tested was that during lockdown young males in particular would engage in more frequent gambling online compared to their previously reported gambling behaviours.

\section{Methods}

ALSPAC is an intergenerational longitudinal cohort study that recruited pregnant women residing in Avon, UK with expected dates of delivery 1st April 1991 to 31st December 1992. The initial cohort consisted of 14,541 pregnancies resulting in 14,062 live births and 13,988 children who were alive at 1 year of age. From the age of seven onwards, the initial sample was bolstered with eligible cases who had originally failed to join the study and there were subsequently 14,701 children alive at 1 year of age following this further recruitment (Boyd et al., 2013). An update on the index children in ALSPAC has recently been published (Northstone et al., 2019). The study website contains details of all the data 
that are available through a fully searchable data dictionary and variable search tool (http:// www.bristol.ac.uk/alspac/researchers/our-data/).

ALSPAC participants were sent two extra online questionnaires during lockdown- the first in April 2020 and the second at the end of May 2020. The questionnaires were developed and deployed using REDCap (Research Electronic Data CAPture tools (Harris et al., 2009); a secure web application for building and managing online data collection exercises, hosted at the University of Bristol.

The gambling questions were included in the second questionnaire, the details of which have been published as a Data note (Northstone et al., 2020). The second online questionnaire, asking about physical and mental health, lifestyle and behaviours, employment and finance, was deployed across the parent and offspring generations between the 26th May and 5th July 2020. The response rate was 44\%, resulting in 2711 young adults completing the questionnaire.

The gambling questions were similar to those previously asked to the same cohort when aged 24 years (Hollén et al., 2020), which were originally derived from the British Gambling Prevalence Survey (Wardle et al., 2008). However, whereas previous gambling questionnaires included 17 questions on what types of gambling activities participants took part in, the COVID questionnaire was reduced to 8 questions about participation in last month with regards to: national lottery, scratch cards, online gambling, online betting, betting exchange, spread betting, private betting, other form of gambling (Appendix). Response options were: not at all, less than weekly, every week or every day/almost every day. Occasional gamblers were defined as those who gambled less than weekly, and regular gamblers as those who gambled weekly or more frequently.

Questions on mental health and wellbeing included specific questions about worries during the pandemic. Depression was assessed using the Short Moods and Feelings Questionnaire (SMFQ) (Ancold \& Stephen, 1995) and anxiety using the General Anxiety Disorder-7 questionnaire (GAD-7) (Spitzer et al., 2006). Participants' wellbeing was selfreported using the Warwick-Edinburgh Mental Wellbeing Scales (WEMWBS) (Tennant et al., 2007). Alcohol use was self-reported with the AUDIT Tool (Bush et al., 1998).

Employment status during lockdown was reported in the following categories: "working/studying same or more hours", "working/studying reduced hours/on paid leave/on furlough", "not in paid work/unpaid leave", "self-employed currently working", "selfemployed not currently working". Participants were asked about how they managed financially pre-COVID and reported as "living comfortably", "doing all right", "just about getting by" and "finding it quite/very difficult".

\section{Consent}

Completion of the lockdown questionnaires was optional and choosing to complete the questionnaire is considered informed consent for the questionnaire. Ethical approval for the study was obtained from the ALSPAC Ethics and Law Committee and the Local Research Ethics Committees. Informed consent for the use of data collected via questionnaires and clinics was obtained from participants following the recommendations of the ALSPAC Ethics and Law Committee at the time. Study participants have the right to withdraw their consent for elements of the study or from the study entirely at any time. Full details of the ALSPAC consent procedures are available on the study website (http://www.bristol.ac.uk/ alspac/researchers/research-ethics/). 


\section{Statistical Analyses}

All analyses were conducted using Stata v.15.1. Chi-square tests, two-proportion z-tests, ANOVAs, binary logistic regressions or multinomial logistic regressions were used.

\section{Results}

\section{Sample}

The second ALSPAC COVID-19 questionnaire was complete by 2711 young adults, with a mean age of 27.8 years (SD: 0.6). The population who responded were predominantly white $(>96 \%)$ and $80 \%$ had at least A-level qualifications. Of these respondents, 2632 (97.1\%) answered the gambling questions: 771 males (29.3\%) and $1861(70.7 \%)$ females.

Previously 4304 ALSPAC participants, with a mean age of 24.9 years (SD: 0.6), had answered the 24-year gambling questionnaire: 1501 males (34.9\%) and $2803(65.1 \%)$ females. The female preponderance was more marked in the respondents to the COVID-19 questionnaire than in the 24 year sample $(\mathrm{p}<0.001)$. The bias introduced by this female preponderance in the COVID-19 questionnaire probably resulted in an underestimate of the number of regular gamblers.

Of those individuals that answered the gambling questionnaire at 24 years, $(\mathrm{N}=4304)$, 3872 (90\%) were sent the COVID questionnaire but only $2160(56 \%)$ answered the questionnaire. Those that did not answer the COVID questionnaire were more likely to be male, have a lower IQ (measured at age 8), have slightly raised hyperactivity scores (measured at age 16.5), were more likely to have a diagnosis of moderate/severe alcohol use disorder (measured at age 22) and more likely to have smoked 100+ cigarettes in their life time (measured at age 25). There was no influence on whether the questionnaire was answered or not of regular gambling at age 24 , employment status at age 20 , or if there was a history of maternal gambling.

\section{Gambling Frequency}

The frequency of gambling during the COVID-19 lockdown, compared with the frequency previously reported at 24 years, is presented in Table 1. Overall, gambling frequency reduced during lockdown for both males and females ( $<$ weekly and $>=$ weekly: $p<0.001$ both sexes). The gender difference widened in those who engaged in regular gambling, with males nearly 3 times more likely than females to gamble regularly.

Table 1 Gambling Frequency by ALSPAC participants during COVID lockdown and at age 24 years

\begin{tabular}{llllll}
\hline & \multicolumn{2}{l}{ COVID-19 questionnaire } & & \multicolumn{2}{l}{ 24-year questionnaire } \\
\cline { 2 - 3 } \cline { 5 - 6 } \cline { 5 - 6 } & Males $(\mathrm{n}=771)$ & Females $(\mathrm{n}=1861)$ & & Males $(\mathrm{n}=1501)$ & Females $(\mathrm{n}=2803)$ \\
\hline No gambling & $614(79.6 \%)$ & $1693(91.0 \%)$ & & $490(32.6 \%)$ & $1202(42.9 \%)$ \\
$<$ weekly gambling & $91(11.8 \%)$ & $115(6.2 \%)$ & & $773(51.5 \%)$ & $1391(49.6 \%)$ \\
\hline$=$ weekly gambling & $66(8.6 \%)$ & $53(2.9 \%)$ & & $238(15.9 \%)$ & $210(7.5 \%)$ \\
\hline
\end{tabular}


Gambling at 24 years strongly predicted whether people gambled or not during lockdown irrespective of gender (OR $(95 \% \mathrm{CI}): 10.70(6.57,17.44), \mathrm{n}=2112)$. Using the subsample of individuals who gambled at both times $(n=1255)$ showed that a larger proportion of males compared to females did not change their frequency of gambling $(\mathrm{p}<0.001)$ whereas a larger proportion of females decreased their gambling frequency during lockdown $(\mathrm{p}<0.001)$. The proportion that increased their gambling frequency did not differ between males and females $(\mathrm{p}=0.28$; Table 2$)$.

\section{Gambling Activities}

The different gambling activities reported in the 24 year and COVID questionnaires are presented in Table 3, which shows a different pattern for regular (weekly or more frequent) gamblers compared to occasional (less than weekly) gamblers. For regular gamblers, a few activities, including national lottery, private betting and online gambling, increased during COVID-19 lockdown compared to what was reported 3 years previously. The proportion gambling on other activities remained stable. In contrast, for occasional gamblers, most gambling activities decreased in frequency or remained stable, with the exception of online gambling which increased in frequency for both occasional and regular gamblers. Online gambling included online poker, bingo, casino games. Online betting, involving placing bets on any event or sport, including e-sports, overall remained at similar rates to 24 years (although most UK based sporting events were cancelled during the lockdown).

\section{Gambling and Mental Health}

Anxiety levels reported by the cohort during lockdown were generally high (Table 4), but there did not appear to be a relationship between anxiety levels and gambling frequency $\left(\chi^{2}=5.8, \mathrm{p}=0.45\right)$.

Around $15 \%$ of participants reported depressive symptoms during lockdown (Table 5), but likelihood of depression was not associated with gambling frequency $\left(\chi^{2}=1.4\right.$, $\mathrm{p}=0.50)$.

Wellbeing scores (mean [SD]) during lockdown were similar in non-gamblers (44 [8.5]), occasional gamblers (43 [8.8]), and regular gamblers (44 [8.7]; $p=0.20)$.

\section{Gambling and Alcohol Use}

Although frequency of drinking any alcohol (<weekly or $>=$ weekly) during lockdown did not show an association with gambling frequency $(\mathrm{p}=0.38)$, frequency of heavy drinking did $(\mathrm{p}<0.001)$. Participants drinking more than 6 units on one occasion regularly (weekly or more) were more likely to be male [OR $(95 \% \mathrm{CI}): 1.62(1.31,2.01)]$ and

Table 2 Correlation of gambling frequency at 24 years and during COVID lockdown

\begin{tabular}{llll}
\hline Frequency during COVID & Males & Females & Total \\
\hline Decreased frequency & $303(77.9 \%)$ & $746(86.1 \%)$ & $1049(83.6 \%)$ \\
Stable frequency & $64(16.5 \%)$ & $83(9.6 \%)$ & $147(11.7 \%)$ \\
Increased frequency & $22(5.7 \%)$ & $37(4.3 \%)$ & $59(4.7 \%)$ \\
Totals & $389(100 \%)$ & $866(100 \%)$ & 1255 \\
\hline
\end{tabular}


Table 3 Gambling Activities reported in the COVID-19 and 24-year questionnaires

\begin{tabular}{|c|c|c|c|}
\hline & \multicolumn{3}{|c|}{ Gambling frequency } \\
\hline & Not at all & $<$ weekly & $>=$ weekly \\
\hline \multicolumn{4}{|l|}{ National lottery } \\
\hline COVID $(n=320)$ & $145(45.3 \%)$ & $106(33.1 \%)$ & $69(21.6 \%)$ \\
\hline Age $24(n=2607)$ & $939(36.0 \%)$ & $1487(57.0 \%)$ & $181(6.9 \%)$ \\
\hline P-value & 0.001 & $<0.001$ & $<0.001$ \\
\hline \multicolumn{4}{|l|}{ Scratch cards } \\
\hline COVID $(n=316)$ & $206(65.2 \%)$ & $90(28.5 \%)$ & $20(6.3 \%)$ \\
\hline Age $24(n=2605)$ & $904(34.7 \%)$ & $1568(60.2 \%)$ & $133(5.1 \%)$ \\
\hline P-value & $<0.001$ & $<0.001$ & 0.36 \\
\hline \multicolumn{4}{|l|}{ Online gambling } \\
\hline COVID $(n=320)$ & $215(67.2 \%)$ & $74(23.1 \%)$ & $30(9.7 \%)$ \\
\hline Age $24(n=2579)$ & $2235(86.7 \%)$ & $310(12.0 \%)$ & $34(1.3 \%)$ \\
\hline P-value & $<0.001$ & $<0.001$ & $<0.001$ \\
\hline \multicolumn{4}{|l|}{ Online betting } \\
\hline COVID $(n=321)$ & $221(68.9 \%)$ & $78(24.3 \%)$ & $22(6.8 \%)$ \\
\hline Age $24(n=2594)$ & $1798(69.3 \%)$ & $651(25.1 \%)$ & $145(5.6 \%)$ \\
\hline P-value & 0.87 & 0.76 & 0.36 \\
\hline \multicolumn{4}{|l|}{ Betting exchange } \\
\hline COVID $(n=319)$ & $313(98.1 \%)$ & $<5(1.3 \%)$ & $<5(0.6 \%)$ \\
\hline Age $24(n=2585)$ & $2476(95.8 \%)$ & $96(3.7 \%)$ & $13(0.5 \%)$ \\
\hline P-value & 0.04 & 0.02 & $<0.001$ \\
\hline \multicolumn{4}{|l|}{ Spread betting } \\
\hline COVID $(n=319)$ & $314(98.4 \%)$ & $<5(0.9 \%)$ & $<5(0.6 \%)$ \\
\hline Age $24(n=2579)$ & $2533(98.2 \%)$ & $42(1.6 \%)$ & $<5(0.2 \%)$ \\
\hline P-value & 0.78 & 0.35 & 0.08 \\
\hline \multicolumn{4}{|l|}{ Private betting } \\
\hline COVID $(n=322)$ & $279(86.6 \%)$ & $35(10.9 \%)$ & $8(2.5 \%)$ \\
\hline Age $24(n=2586)$ & $2083(80.5 \%)$ & $490(19.0 \%)$ & $13(0.5 \%)$ \\
\hline P-value & 0.008 & $<0.001$ & $<0.001$ \\
\hline \multicolumn{4}{|l|}{ Other gambling } \\
\hline COVID $(n=322)$ & $303(94.1 \%)$ & $10(3.1 \%)$ & $9(2.8 \%)$ \\
\hline Age $24(n=2559)$ & $2489(97.3 \%)$ & $60(2.3 \%)$ & $10(0.4 \%)$ \\
\hline P-value & 0.002 & 0.40 & $<0.001$ \\
\hline
\end{tabular}

Sample size represents those that said they have gambled on one or more activities

2.38 times (CI: $1.56,3.63)$ more likely to gamble weekly during lockdown compared to not gambling at all. The odds ratio for less than weekly gambling was $1.83(1.30,2.56)$, no different to that for weekly gambling $(\mathrm{p}=0.31)$. The association between heavy alcohol intake and less than weekly gambling was only significant in males [2.67 (1.66, $4.30)$; females: $1.04(0.61,1.78)]$, whereas the association with weekly or more frequent gambling was evident in both sexes [males: 2.03 (1.15, 3.58); females: 2.42 (1.28, 4.61)]. 


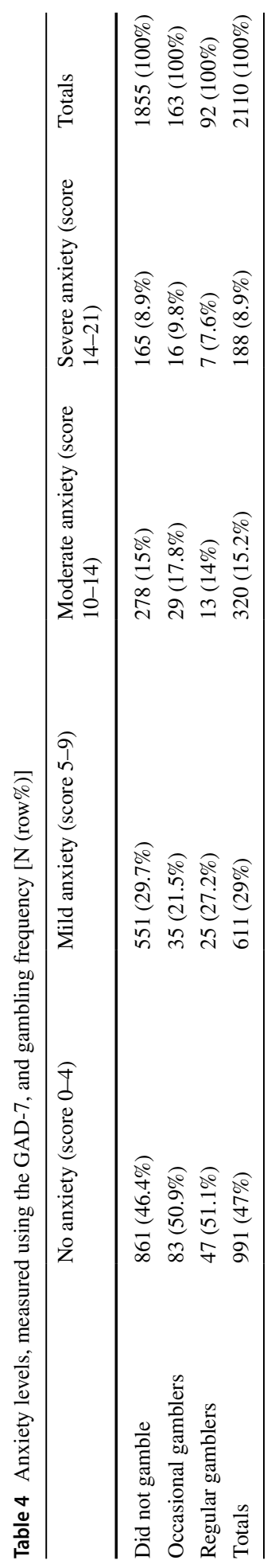


Table 5 Probability of depression, measured on the SMFQ, and gambling frequency

\begin{tabular}{llll}
\hline & $\begin{array}{l}\text { Unlikely depression } \\
(\text { score }<12)\end{array}$ & $\begin{array}{l}\text { Likely depression (score } \\
12+)\end{array}$ & Totals \\
\hline Did not gamble & $1553(84.6 \%)$ & $283(15.4 \%)$ & $1836(100 \%)$ \\
Occasional gamblers & $133(82 \%)$ & $29(17.9 \%)$ & $162(100 \%)$ \\
Regular gamblers & $78(87.6 \%)$ & $11(12.4 \%)$ & $89(100 \%)$ \\
Totals & $1764(84.5 \%)$ & $323(15.5 \%)$ & $2087(100 \%)$ \\
\hline
\end{tabular}

\section{Gambling and Personal Financial Situation}

Those that struggled financially before COVID were more likely to answer yes to any gambling during lockdown ( $<$ weekly gambling and $>=$ weekly gambling combined due to small numbers). Odds ratio for those reporting "just getting by" pre-COVID and any gambling was $1.56(95 \% \mathrm{CI}: 1.07,2.26)$ and $2.23(1.23,4.06)$ for those reporting "finding it quite or very difficult".

No relationship was apparent between gambling frequency and current employment status during lockdown $(\mathrm{p}=0.63)$.

\section{Discussion}

This study of a well-characterised group of young adults during COVID-19 mitigation has captured in 'real time' their mood and gambling behavior. As expected, the overall frequency of gambling reduced during lockdown because of the restrictions on movement, but the activity which could be done from home- online gambling- did increase in both sexes. The main hypothesis, that during lockdown young males in particular would engage in more frequent gambling, was supported. Regular gamblers not only gambled online more than previously, but also betted on games at home and gambled on lotteries and using scratchcards more than reported at 24 years. Regular weekly gambling was associated with heavy use of alcohol in both males and females.

The rates of gambling reported in ALSPAC were lower than the British average during lockdown, probably because the sample was $71 \%$ female. During the same period as the ALSPAC COVID-19 questionnaires, YouGov were conducting online surveys during lockdown of 2000 British adults' behaviour, including gambling (YouGov tracker, 2020). The seven waves of the YouGov survey conducted from 16th April to 18th June showed that past-four-week gambling participation by adults of all ages remained relatively stable, within a range of 28-32\%. However, young adults aged 18-34 were significantly more likely than average to report increases in time or money spent on at least one gambling activity. Amongst regular 'engaged' gamblers, YouGov also found an increase in trying new online activities, such as online bingo, betting on virtual races or sports, National Lottery online and online slots.

The increase in online gambling seen in ALSPAC was not surprising considering the circumstances of lockdown, and consistent with the steady increase previously seen in the same respondents from 17 to 24 years in the use of online methods of gambling and betting (Hollén et al., 2020). As the increase in home working may well continue after the pandemic, it also possible that gambling online may continue to increase as a recreational activity for young adults spending more time at home. Gaming and 
gambling online are showing increasing convergence all over the world (Kim \& King, 2020). The COVID-19 pandemic is likely to exacerbate existing inequalities that are already expressed in the distribution of gambling harms: a public health approach to the reduction of gambling related harms is called for (Griffiths et al 2020).

Data from the biggest gambling operators in the UK, covering $80 \%$ of the online gambling market (Gambling Commission, 2020), show that gross gambling yield increased during lockdown for online betting and online poker, but the biggest change was in betting online on e-sports, the income from which increased $3000 \%$ during the lockdown period between March-June 2020. Betting on e-sports was not separated in the ALSPAC COVID-19 questionnaire, but undoubtably increased as the usual outlets for sports betting (e.g. soccer) were suspended. A review of player data from a large European operator showed a decrease in the amount of money wagered by sports bettors during the COVID-19 pandemic (compared with before it) and that sports bettors did not switch to playing more online casino games. (Auer et al., 2020). In Sweden, those reporting sports betting during a period with decreased sports betting opportunities were shown to have markedly higher gambling problems (Håkansson, 2020). An online survey of regular sports betters in the UK during COVID lockdown (Wardle et al., 2021) has demonstrated that, while betting generally decreased, problem gambling was associated with starting new gambling activities during lockdown for men and that women who were shielding for health reasons were especially vulnerable to gambling harms.

The association between regular gambling and heavy regular alcohol use seen during lockdown has previously been observed in ALSPAC at 24 years (Hollén et al., 2020), and has been widely reported in the gambling literature (e.g. Scholes-Balog et al., 2016). The ALSPAC sample size was too small to look at alcohol intake change between age 24 years and during lockdown. The lack of association with mental health and well-being was surprising given the alcohol link, but previous studies have suggested that gambling and mood have a complex bi-directional relationship in young people (Chinneck et al., 2016; Dussault et al 2011).

The frequency of gambling during COVID-19 lockdown did not show any clear relationship with employment status: this may reflect the lockdown sample being well educated, as at the 24 year assessment, regular gambling in ALSPAC was associated with not being in education or employment (Hollén et al., 2020). The Health Survey for England in 2018 (NHS Digital, 2019) reported for adults over 16 years the following frequencies of any gambling by economic activity: employed $62 \%$, retired $50 \%$, in full time education $26 \%$, unemployed $41 \%$, other inactive $39 \%$.

The gamblers in the ALSPAC study did report more financial difficulties pre- lockdown than non-gamblers, and some young people may have been gambling during lockdown in response to, or to escape from, financial hardship. However, the questionnaire data were not detailed enough to conclude any relationship with gambling spend.

The strengths of this study were the use of a well characterised cohort of young adults, some of whom had answered the same gambling questions 3 years previously, with contemporaneous self- report of mental health and well-being. The study also had some important limitations: although the sample size was large it was a self-selected, well-educated sub-group of the wider ALSPAC cohort; the majority of respondents were female, whereas males were more likely to be regular gamblers. Consequently, the associations shown were likely to underestimate the true extent of gambling activity during lockdown. 


\section{Conclusions}

Gambling online saw the biggest increase during lockdown, irrespective of gambling frequency. In regular gamblers, the proportion playing national lottery and doing private betting also increased but most other gambling activities remained stable or, for occasional gamblers, decreased in frequency. Previous gambling at aged 24, a history of financial difficulties pre-COVID, and heavy regular use of alcohol, were associated with regular gambling during lockdown.

\section{Appendix}

See Table 6. 


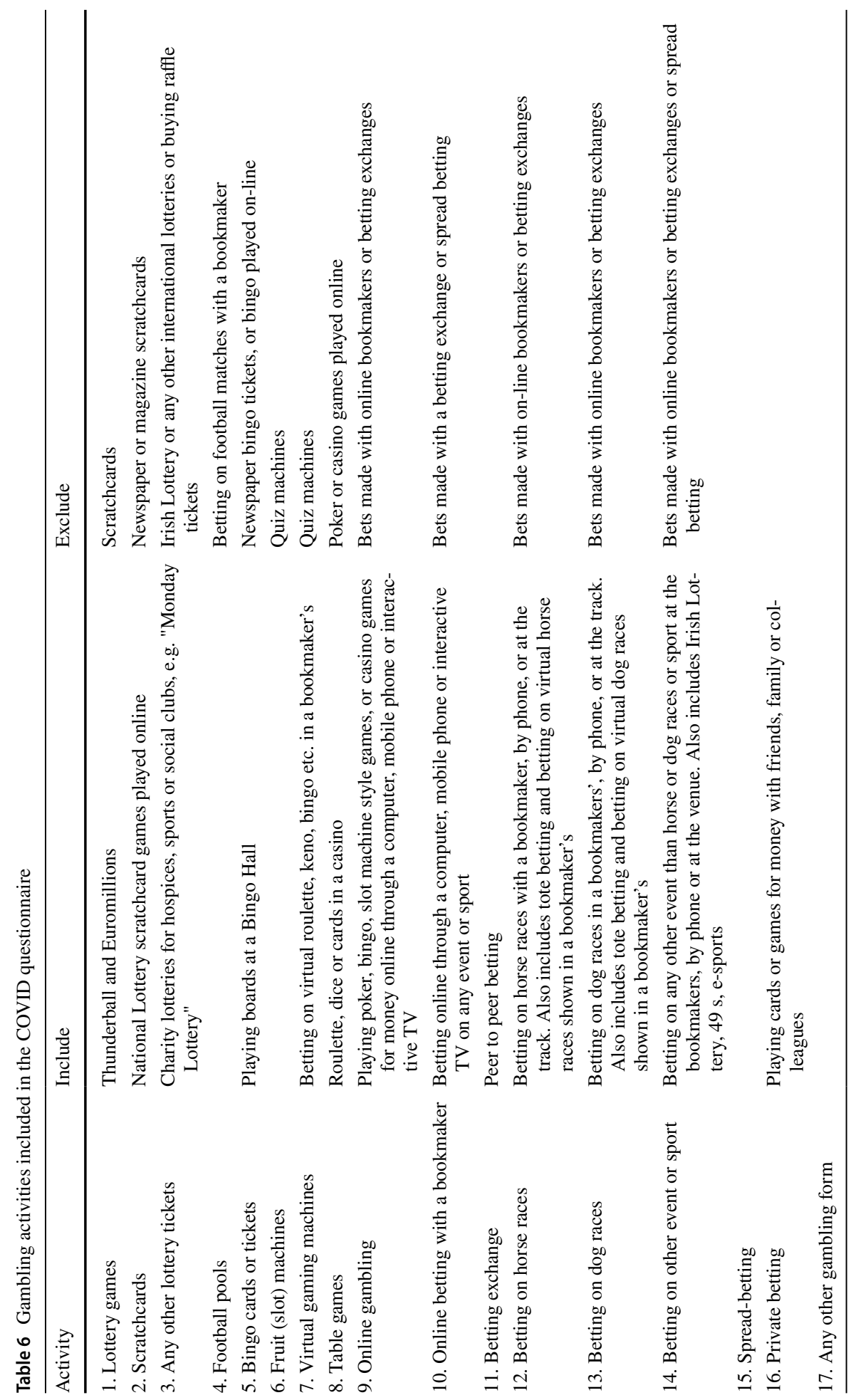


Acknowledgements We are extremely grateful to all the families who took part in this study, the midwives for their help in recruiting them, and the whole ALSPAC team, which includes interviewers, computer and laboratory technicians, clerical workers, research scientists, volunteers, managers, receptionists and nurses. Study data were collected and managed using REDCap electronic data capture tools hosted at the University of Bristol.

Funding The UK Medical Research Council and the Wellcome Trust (Grant ref: 102215/2/13/2) and the University of Bristol provide core support for the ALSPAC. A comprehensive list of grant funding is available on the ALSPAC website (www.bris.ac.uk/alspac). Specific funding for the ALSPAC Gambling Study was supplied by GambleAware (formerly the Responsible Gambling Trust) and the University of Bristol. The collection of data during lockdown using the ALSPAC COVID 19 questionnaires were funded by the Wellcome Trust.

\section{Declaration}

Conflicts of interest The authors do not have any conflicts to declare.

Open Access This article is licensed under a Creative Commons Attribution 4.0 International License, which permits use, sharing, adaptation, distribution and reproduction in any medium or format, as long as you give appropriate credit to the original author(s) and the source, provide a link to the Creative Commons licence, and indicate if changes were made. The images or other third party material in this article are included in the article's Creative Commons licence, unless indicated otherwise in a credit line to the material. If material is not included in the article's Creative Commons licence and your intended use is not permitted by statutory regulation or exceeds the permitted use, you will need to obtain permission directly from the copyright holder. To view a copy of this licence, visit http://creativecommons.org/licenses/by/4.0/.

\section{References}

Ancold, A. D. R. I. A. N., \& Stephen, C. (1995). Development of a short questionnaire for use in epidemiological studies of depression in children and adolescents. Age (years), 6(11), 237-249

Auer, M., Malischnig, D., \& Griffiths, M. D. (2020). Gambling before and during the COVID-19 pandemic among European regular sports bettors: An empirical study using behavioral tracking data. International Journal of Mental Health and Addiction, 1-8

Boyd, A., Golding, J., Macleod, J., Lawlor, D. A., Fraser, A., \& Davey Smith, G. (2013). Cohort profile: The 'Children of the 90s'-The index offspring of the Avon Longitudinal Study of Parents and Children. International Journal of Epidemiology, 42, 111-127

Bray, B. C., Lee, G. P., Liu, W., Storr, C. L., Ialongo, N. S., \& Martins, S. S. (2014). Transitions in gambling participation during late adolescence and young adulthood. Journal of Adolescent Health, 55(2), 188-194

Bush, K., Kivlahan, D. R., McDonell, M. B., et al. (1998). The AUDIT alcohol consumption questions (AUDIT-C): An effective brief screening test for problem drinking. Ambulatory Care Quality Improvement Project (ACQUIP) alcohol use disorders identification test. Archives of Internal Medicine, 158(16), 1789-1795

Calado, F., Alexandre, J., \& Griffiths, M. D. (2017). Prevalence of adolescent problem gambling: A systematic review of recent research. Journal of Gambling Studies, 33(2), 397-424

Chinneck, A., Mackinnon, S. P., \& Stewart, S. H. (2016). Investigating possible reciprocal relations between depressive and problem gambling symptoms in emerging adults. The Canadian Journal of Psychiatry, 61(2), 93-101

Delfabbro, P., King, D., \& Griffiths, M. D. (2014). From adolescent to adult gambling: An analysis of longitudinal gambling patterns in South Australia. Journal of Gambling Studies, 30, 547-563

Dussault, F., Brendgen, M., Vitaro, F., Wanner, B., \& Tremblay, R. E. (2011). Longitudinal links between impulsivity, gambling problems and depressive symptoms: A transactional model from adolescence to early adulthood. Journal of Child Psychology and Psychiatry, 52, 130-138

Emond, A., Griffiths, M. D., \& Hollén, L. (2020). Problem gambling in early adulthood: A populationbased study. International Journal of Mental Health and Addiction, 1-17 
French, M. T., Maclean, J. C., \& Ettner, S. L. (2008). Drinkers and bettors: Investigating the complementarity of alcohol consumption and problem gambling. Drug and Alcohol Dependence, 96(1-2), $155-164$

Gambling Commission 2019: Young people and gambling. Retrieved from https://www.gamblingco mmission.gov.uk/PDF/Young-People-Gambling-Report-2019.pdf

Gambling Commission 2020: Gambling business data on gambling during Covid-19, updated October 2020. Retrieved from https://www.gamblingcommission.gov.uk/news-action-and-statistics/Stati stics-and-research/Covid-19-research/Covid-19-updated-October-2020/Gambling-business-dataon-gambling-during-Covid-19-updated-October-2020.aspx

Griffiths, S., Reith, G., Wardle, H., \& Mackie, P. (2020). Pandemics and epidemics: public health and gambling harms. Public Health, 184, 1

Håkansson, A. (2020). Impact of COVID-19 on online gambling-a general population survey during the pandemic. Frontiers in Psychology, 11, 2588

Harris, P. A., Taylor, R., Thielke, R., Payne, J., Gonzalez, N., \& Conde, J. G. (2009). Research electronic data capture (REDCap) - A metadata-driven methodology and workflow process for providing translational research informatics support. Journal of Biomedical Informatics, 42(2), 377-381

Hollén, L., Dörner, R., Griffiths, M. D. \& Emond, A. (2020). Gambling in young adults aged 17-24 years: A population-based study. Journal of Gambling Studies, 36(3), 747-766

Killick, E. A., \& Griffiths, M. D. (2019). In-play sports betting: A scoping study. International Journal of Mental Health and Addiction, 17, 1456-1495

Kim, H. S., \& King, D. L. (2020). Gambling-gaming convergence: new developments and future directions. International Gambling Studies, 1-7

NHS Digital. (2019). Health Survey for England 2018, supplementary analysis on gambling. Retrieved from https://digital.nhs.uk/data-and-information/publications/statistical/health-survey-for-england/ 2018/health-survey-for-england-2018-supplementary-analysis-on-gambling

Northstone, K., Lewcock, M., Groom, A., Boyd, A., Macleod, J., Timpson, N. J., \& Wells, N. (2019). The Avon Longitudinal Study of Parents and Children (ALSPAC): An update on the enrolled sample of index children in 2019. Wellcome Open Research, 4, 51

Northstone, K., Smith, D., Bowring, C., Wells, N., Crawford, M., Haworth, S., \& Timpson, N. J. (2020). The Avon Longitudinal Study of Parents and Children-A resource for COVID-19 research: Questionnaire data capture May-July 2020. Wellcome Open Research, 5.

Petry, N. M., \& Weinstock, J. (2007). Internet gambling is common in college students and associated with poor mental health. American Journal on Addictions, 16(5), 325-330

Scholes-Balog, K. E., Hemphill, S. A., Toumbourou, J. W., \& Dowling, N. A. (2016). Problem gambling patterns among Australian young adults: Associations with prospective risk and protective factors and adult adjustment outcomes. Addictive Behaviors, 55, 38-45

Spitzer, R. L., Kroenke, K., Williams, J. B. W., et al. (2006). A brief measure for assessing generalized anxiety disorder: The GAD-7. Archives of Internal Medicine, 166(10), 1092-1097

Tennant, R., Hiller, L., Fishwick, R., et al. (2007). The Warwick-Edinburgh mental wellbeing scale (WEMWBS): Development and UK validation. Health and Quality of Life Outcomes, 5, 63

Wardle, H., Donnachie, C., Critchlow, N., Brown, A., Bunn, C., Dobbie, F., \& Hunt, K. (2021). The impact of the initial Covid-19 lockdown upon regular sports bettors in Britain: Findings from a cross-sectional online study. Addictive Behaviors, 118, 106876

Wardle, H., Sproston, K., Orford, J., Erens, B., Griffiths, M. D., Constantine, R., \& Pigott, S. (2008). The British gambling prevalence survey 2007. National Centre for Social Research. Retrieved from: https:// www.tsoshop.co.uk/product/9780117037922/British-gambling-prevalence-survey-2007

YouGov Covid-19 tracker (2020). https://github.com/YouGov-Data/covid-19-tracker

Publisher's Note Springer Nature remains neutral with regard to jurisdictional claims in published maps and institutional affiliations. 